\title{
Mutagenesis at Five Pteridine Pathway Loci in Drosophila melanogaster: Genetic and Biochemical Characterization
}

\author{
J.M. Millán and C. Nájera ${ }^{\$}$ \\ Departmento de Genetica, Facultad de Ciencias Biológicas, Dr. Moliner 50, 46100 Burjassot, \\ Valencia, Spain \\ (Received March 20, 1993)
}

\section{Summary}

In order to carry out a genetic and biochemical analysis of pteridin pathway eye colour loci, sixteen strains of five cye colour mutants of Drosophila melanogaster ( $2 \mathrm{dkc}, 7 \mathrm{sf}, 3 \mathrm{se}, 3 \mathrm{Hn}^{\mathrm{r}}$ and 1 bw) from natural populations were used. Four EMS mutagenesis experiments were carried out to produce induced mutants of the same loci. 54 mutants (mosaics and completes) were obtained but only 7 (4 sf, 1 bw, $1 \mathrm{Hn}^{\mathrm{r}}$ and $1 \mathrm{dke}$ ) could be isolated. The $40.48 \%$ of the mutations were mosaics. The percentage of mutants appeared during the four first days $(85.19 \%)$ was significatively higher to the percentage of mutants appeared luring the four following $(14.18 \%)$. Viabilities of EMS-induced mutants were similar to that of the natural ones. For the induced mutants viability at $25^{\circ} \mathrm{C}$ was higher than at $16^{\circ} \mathrm{C}$ and heterozygotes had a higher viability than mutant homozygotes. The low mutagenesis frequencies and the lack of some metabolites for $\mathrm{Hn}^{\mathrm{r}}$ and se mutants suggest an important role of these mutants in the pteridine pathway.

Key words: Mutagenesis, Eyc color mutants, Pteridines, Drosophila melanogaster

\section{Introduction}

Two classes of pigments contribute to the final colour of Drosophila eyes: the ommochromes and the pteridines. The ommochromes pathway was established long time ago, however, the pathways for the biosynthesis of the red pigments still remain incomplete at present. A hypothetical pathway (Fig. 1). was proposed (1). The only loci which are sure involved as structural genes in this pathway are Punch (GTP cyclohydrolase enzyme), purple (sepiapterine sintase A enzyme) and rosy (xantin dehydrogenase enzyme). The study of eye colour mutants in Drosophila means an advance in the knowledge of the pathway, nevertheless the location of structural genes is difficult principally due to pleiotropic effects of genes and sometimes to lethality. In the present work. five pteridine pathway mutants were used: 3 of them located in the second chromosome

$\S$ Author to whom correspondence should be addressed. of Drosophila melanogaster (dark-eye, safranin and brown) and the other two located in the third one (Henna-recessive and sepia). The function of these five mutants in the pathway is unknown but Ferré et al. (2) proposed the hypothesis that $\mathrm{Hn}^{\mathrm{r}}$ blocked the formation of tetrahydropterin and se the formation of acetyldihydrohomopterin (Fig. 1). Enviromental mutagenesis experiments were carried out in order to get induced mutants. The Ethyl methanesulfonate (EMS) was chosen among all the chemical mutagens because its water solubility, stability effectiveness and easy handling (3-6). A genetical and biochemical study of natural and induced mutants of the five loci was made in order to contribute to understand the role of these mutants in the pteridin pathway.

\section{Materials and Methods}

Drosophila strains and culture 


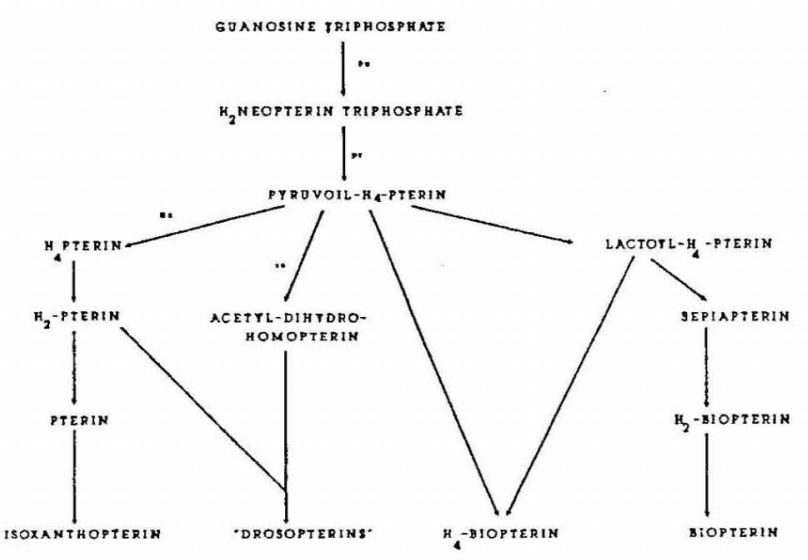

Figure 1. Proposed pathway for pteridine biosynthesis in Drosophila melanogaster.

Sixteen strains of five eye colour mutants of Drosophila melanogaster ( 2 dke, 7 sf, 3 se, $3 \mathrm{Hn}^{r}$ and $1 \mathrm{bw})$ from natural populations where used. These strains were obtained by Nájera and Ménsua (7) from a total of six captures from three different habitats; a cellar in Requena (Valencia, Spain) a vineyard in E1 Portón (4 km away from the cellar) and a semi built up pincwood at La Cañada $(70 \mathrm{~km}$ away from Requena) on spring and autumn in 1981. Marker strains used to obtain EMS-induced mutants were al b en sp homozygous males for the 2nd chromosome and st ri ss homozygous for the 3rd one; also, standard mutant females for each one of the five eye colour mutations were used to be crossed by the mutagenized males except for bw. For this locus the deficient strain $\operatorname{Df}(2 \mathrm{R}) \mathrm{bw}^{5}, \mathrm{bw}^{5} / \operatorname{In}(2 \mathrm{~L})$ $\mathrm{Cy}, \mathrm{dp}^{\mathrm{j} \mathrm{v} 2}$ was used. The strains were obtained from the collection in Bowling Green (USA) except st ri ss which was set up by introducing the st mutant in the ri ss strain. All strains were maintained at $25 \pm 1^{\circ} \mathrm{C}, 60 \pm 10 \%$ relative humidity under constant lighting, in $125 \mathrm{ml}$ bottles with $30 \mathrm{ml}$ of a standard medium of corn flour, agar-agar, sugar and liver yeast.

\section{Mutagenesis}

Four mutagenesis experiments were carried out by feeding EMS to Drosophila melanogaster males, homozygous for second chromosomes marked with al b cn sp or for third marked with st ri ss. Feeding method was chosen because its power in producing punctual mutations without affecting development is higher than inhalation or injection methods. Only males were fed because of the higher mutagenesis action of EMS on spermatocytes than on oocytes.
So, three day-old males were fed in $40 \mathrm{mM}$ EMS solution in 5\% sucrose for 24 hours at room temperature (8). The mutagenized males were allowed 24 hours to recover. Each male was placed in a vial containing standard medium and crossed $6 / 8$ virgin mutant females, homozygous for the standard allele of one of the five studied mutations. Males were transferred to fresh virgin females, every two days, each transfer constituting a brood. This type of brood analysis allows to sample premeiotic, meiotic and postmetiotic sperm cells. The first three broods (0 to 6 days) sample postmeiotic sperm cells, the fourth brood samples primarily meiotic cells and the last broods sample premeiotic cells. The induced mutants were isolated from mass $F_{1}$ cultures according to the following scheme:

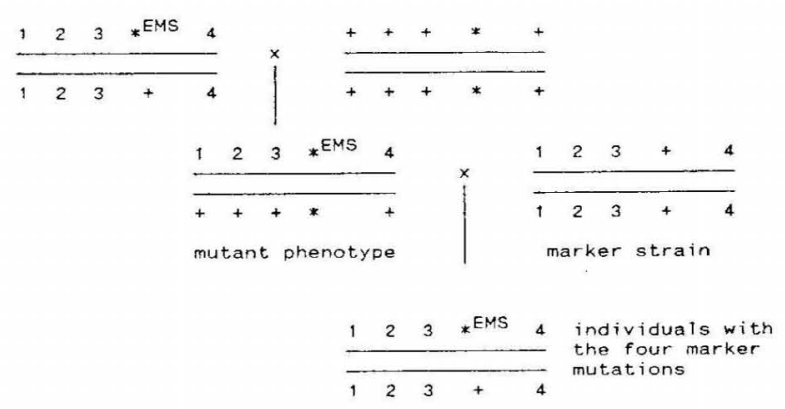

where $*=$ one of the five standard mutants (sf, se, bw, $\mathrm{Hn}^{\mathrm{r}}$, dke) 1, 2, 3, 4=marker mutations.

By inbreeding, individuals with homozygous mutant phenotype

$$
\frac{123 *^{\text {EMS }} 4}{123 *^{\text {EMS }} 4}
$$

were selected from the progeny and the EMS-induced strain was set up.

\section{Complementation analysis}

Reciprocal crosses of all pairwise combinations of natural and induced mutants were carried out at $25^{\circ} \mathrm{C}$ and $800 / 1000$ progeny were scored.

\section{Viability and developmental time}

For viability testing flies were left for a maximum of 12 hours in the usual medium (9) and afterwards the eggs were collected. One hundred eggs were placed in each vial and ten repetitions were made for each case. The number of emerged adults was counted. For developmental time testing, the formula $\left(\Sigma_{\mathrm{i}} \mathrm{n}_{\mathrm{i}} \times \mathrm{i} / \mathrm{n}_{1}\right)$ was used. 
where $n_{i}=$ total flies emerged in day $i$

$n_{1}=$ total flies emerged in the vial.

A total of ten repetitions were also made in each case. Viability and developmental time of EMS-induced mutants were analysed at two different temperatures, $25^{\circ}$ and $16^{\circ} \mathrm{C}$. These paramethers were also studied in all the heterozygous combinations of the induced mutants with the wild strain Or-R.

\section{Biochemical procedures}

The procedure of Ferré et al. (2) was used to obtain the chromatographic pattern of each EMS-induced mutant with a modification: the preparation of the samples consisted of homogeinizing 20 female heads, 15 male heads and 5 male bodies together. Quantitative estimation of the red eye pigments from the induced mutants was carried out following the procedure of Real et al. (10) and quantitative estimation of isoxanthopterin in these mutants was carried out according the method of Silva and Ménsua (11). In order to improve visual resolution of pteridine levels, we removed ommochrome pigments from eye by including the $\mathrm{cn}$ or st mutations in the marker strains.

\section{Results}

Toxicity test

Assuming the importance of LC50 (Lethal concentration for $50 \%$ of the individuals) in order to know the equilibrium between mutagenicity and mortality, toxicity curves were made and a LC50 of $36.43 \mathrm{mM}$ was obtained. So, $40 \mathrm{mM}$ EMS solution was used since at lower concentrations the frequency of induced mutations is under the sensitivity of the test.

Mutagenesis experiments
Four mutagenesis experiments were carried out at this EMS concentration. The number of total $F_{1}$ flies screened for each mutation (grouping the four experiments together), the number of mosaics obtained for each strain, its frequency, the number of total induced mutants and the number of recovered from them, their frequencies and the total mutation rates for each onc of the five loci are shown in Table 1. In total, 54 mutants (mosaics and completes) were obtained but only 7 could be isolated. 4 of them were safranin (sf2MN, sf4MN, sf5MN and sf6MN), 1 Henna-recessive ( $\left.\mathrm{Hn}^{\mathrm{r}} 3 \mathrm{MN}\right), 1$ dark-eye (dke3MN) and 1 brown (bw2MN). Considering these five loci together, the $40,48 \%$ of these mutations were mosaics. Figure 2 shows the order of appearance of the differents mutants through the time. The percentage of mutants appeared during the four first days (85.19\%) is significatively higher to the percentage of mutants appeared during the four following $(14,18 \%)$ (Table 2). This shows that EMS mutagenesis has exclusive action on postmeiotic sperm cells. No cryptic mutants were found for neither of the five mutations.

\section{Complementation test}

The induced mutants were crossed with the natu-

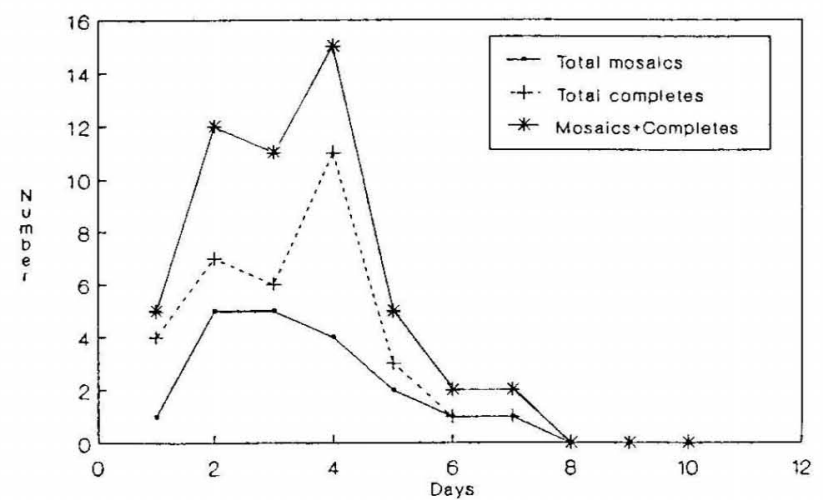

Figure 2. Order of appearance of the induced mutants through the time.

Table 1. Mosaic, complete and total frequencies for each one of the five mutants

\begin{tabular}{|c|c|c|c|c|c|c|c|c|c|}
\hline \multirow{2}{*}{ Mutation } & \multirow{2}{*}{$\begin{array}{c}\mathrm{n}^{\circ} \text { flies } \\
\text { screened }\end{array}$} & \multicolumn{2}{|c|}{ Mosaics } & \multicolumn{4}{|c|}{ Completes } & \multicolumn{2}{|c|}{ Total } \\
\hline & & $\mathrm{n}^{2}$ & freq. & $\mathrm{n}^{\prime \prime}$ & freq. & recov. & lreq. & $\mathrm{n}^{\underline{\underline{e}}}$ & freq. \\
\hline se & 32198 & 1 & $3.1 \times 10^{5}$ & 0 & 0 & 0 & 0 & 1 & $3.1 \times 10^{5}$ \\
\hline $\mathrm{sf}$ & 19654 & 2 & $1.1 \times 10^{-4}$ & 8 & $4.1 \times 10^{-4}$ & 4 & $2.0 \times 10^{-4}$ & 10 & $5.1 \times 10^{4}$ \\
\hline bw & 18654 & $\dot{4}$ & $2.1 \times 10^{-4}$ & 9 & $4.8 \times 10^{4}$ & 1 & $5.3 \times 10^{5}$ & 13 & $6.9 \times 10^{-4}$ \\
\hline $\mathrm{Hn}^{\mathrm{r}}$ & 39947 & 5 & $1.3 \times 10^{-4}$ & 7 & $1.8 \times 10^{4}$ & 1 & $2.5 \times 10^{-5}$ & 12 & $3.0 \times 10^{4}$ \\
\hline dke & 31598 & 7 & $2.2 \times 10^{4}$ & 11 & $3.5 \times 10^{-4}$ & 1 & $3.2 \times 10^{-4}$ & 18 & $5.7 \times 10^{-4}$ \\
\hline
\end{tabular}

treq (frequency): recov (recovered) 
Table 2. Percentage of mutations appeared during the first 4 days versus the subsequent 4

\begin{tabular}{|c|c|c|c|c|c|c|}
\hline \multirow{2}{*}{ Mutation } & \multicolumn{3}{|c|}{ First 4 days } & \multicolumn{3}{|c|}{ Subsequent 4 days } \\
\hline & $\operatorname{mos}$ & com & tot & $\operatorname{mos}$ & com & tot \\
\hline sf & 100.00 & 87.50 & 90.00 & 0 & 12.50 & 10.00 \\
\hline dke & 100.00 & 81.82 & 88.89 & 0) & 18.18 & 11.11 \\
\hline bw & 75.00 & 100.00 & 92.31 & 25.00 & 0 & 7.69 \\
\hline se & 100.00 & () & 100.00 & 0 & 0 & 0 \\
\hline $\mathrm{Hn}^{r}$ & 40.00 & 85.71 & 66.67 & 60.00 & 14.29 & 33.33 \\
\hline Total & 78.95 & 88.57 & 85.19 & 21.05 & 11.43 & 14.81 \\
\hline
\end{tabular}

mos (mosaics): com (complete mutants): tot (totals: mosaics + completes)

ral strains (with the same mutation) and the four induced mutants for sf also among them. In all cases no complementation in the production of the eye colour phenotype has been seen.

\section{Viability and Developmental Time}

An analysis of variance (ANOVA) of the mean viabilities for all mutant strains (natural and induced) was made for each one of the five mutations. The means that do not differ signifiatively were grouped by underlined (Table 3). Viabilities of EMSinduced mutants are similar to that of the natural ones. With regard to the heteroallelic combinations. viability is, in general, higher than viability of the parental strains except for se mutants. A factorial analysis from the sex index indicate variation of viability in relation with the strains and with the sex. In sf, se and bw strains both factors are significant and also its interaction. $\mathrm{In} \mathrm{Hn}^{\mathrm{r}}$ both factors are significant but not the interaction indicating the higher viability of one sex over the other and in dke there are only significant differences among strains and not between sexes. The viability of the induced mutants was higher at $25^{\circ}$ than at $16^{\circ} \mathrm{C}$ except for bw2MN, although in dke3MN and $s f 2$ MN strains differences were not significative (Table 4). Differences in eye colour among the mutants cultured at these two different temperatures were not observed. In all cases heterozygotes had a higher viability than mutant homozygotes although for sf4 MN strain differences were not significative (Table 4). In general, a high variability and a trend to increase the mean developmental time for the induced mutants was observed. With regard to the heteroallelic genotypes, a trend to decrease the mean developmental time with respect to the parental strains for se heteroalleles and a tendency to increase the mean developmental time with respect to the paren-
Table 3. ANOVA of the mean viabilities of natural and induced mutant strains

(Safranin)

\begin{tabular}{|c|c|c|c|c|}
\hline \multicolumn{2}{|l|}{ Source of variation } & SS & MS & \multirow{4}{*}{$\begin{array}{c}\mathrm{F} \\
77.9162^{* * *}\end{array}$} \\
\hline Among groups & 10 & 10.3222 & 1.0332 & \\
\hline Within groups & 99 & 1.3286 & 0.0134 & \\
\hline Total & 109 & 11.6508 & & \\
\hline Mean & Strain & & & \\
\hline-0.5381 & 19BO I & & & \\
\hline-0.3740 & $23 \mathrm{BP}$ & & & \\
\hline-0.3297 & $51 \mathrm{BO}$ & & & \\
\hline-0.2170 & $2 \mathrm{MN}$ & & & \\
\hline-0.1171 & $2 \mathrm{PO}$ & & & \\
\hline 0.0675 & $17 \mathrm{VO}$ & & & \\
\hline 0.0839 & $5 \mathrm{MN}$ & & & \\
\hline 0.1574 & $6 \mathrm{MN}$ & & & \\
\hline 0.2962 & $1 \mathrm{VP}$ & & & \\
\hline 0.3853 & $16 \mathrm{VP}$ & & & \\
\hline 0.4() 46 & $4 \mathrm{MN}$ & & & \\
\hline
\end{tabular}

(Henna Recessive)

\begin{tabular}{lrrrr}
\hline Source of variation & df & \multicolumn{1}{c}{ SS } & MS & F \\
\hline Among groups & 3 & 9.1080 & 3.0360 & $167.8797^{* * * *}$ \\
Within groups & 36 & 0.6510 & 0.0181 & \\
Total & 39 & 9.7590 & & \\
\hline \multicolumn{1}{c}{ Meąn } & Strain & & & \\
-0.9028 & $63 \mathrm{BO}$ । & & & \\
-0.7370 & 28BP । & & & \\
-0.4645 & $3 \mathrm{MN}$ । & & \\
0.3397 & 20VP । & & &
\end{tabular}

(Sepia)

\begin{tabular}{lrccc}
\hline Source of variation & df & SS & MS & F \\
\hline Among groups & 2 & 0.1752 & 0.0876 & $4.0697^{*}$ \\
Within groups & 27 & 0.5811 & 0.0215 & \\
Total & 29 & 0.7563 & & \\
\hline
\end{tabular}

\begin{tabular}{|c|c|}
\hline Mean & Strain \\
\hline 0.1517 & $3 \mathrm{VO} \quad 1$ \\
\hline 0.2890 & $85 \mathrm{BO}$ \\
\hline 0.3305 & $33 \mathrm{BP}$ \\
\hline
\end{tabular}

\begin{tabular}{lrrrr}
\hline Source of variation & df & SS & MS & F \\
\hline Among groups & 2 & 0.8306 & 0.4153 & $6.3681^{* *}$ \\
Within groups & 26 & 1.6956 & 0.0652 & \\
Total & 28 & 2.5262 & & \\
\hline
\end{tabular}

$\begin{array}{lll}\frac{\text { Mean }}{-0.2391} & & \text { Strain } \\ 0.0801 & & 3 \mathrm{BON} \\ 0.1426 & & \text { IBP }\end{array}$


(Brown)

\begin{tabular}{lrrrr}
\hline Source of variation & df & SS & MS & F \\
\hline Among groups & 1 & 0.6553 & 0.6353 & $60.0009^{* * *}$ \\
Within groups & 18 & 0.1996 & 0.0106 & \\
Total & 19 & 0.8259 & & \\
\hline
\end{tabular}

$\mathrm{df}=$ Degrees of freedom

$\mathrm{SS}=$ Sums of squares

$\mathrm{MS}=$ Mean squares

$\mathrm{F}=$ Values of $\mathrm{F}$ test

$*=\mathrm{p}<0.05 * *=\mathrm{p}<0.01 * * *=\mathrm{p}<0.001$

$\mathrm{C}=$ cellar $\mathrm{V}=$ vincyard $\mathrm{P}=$ pine-wood $\mathrm{A}=$ autumn $\mathrm{S}=$ spring $\mathrm{MN}=$ induced mutants

Table 4. Comparison of the viabilities of the induced mutants 1) at two different temperatures $\left(25^{\circ} \mathrm{C}-16^{\circ} \mathrm{C}\right)$ and 2) versus its heterozygotes with Or-R

\begin{tabular}{|c|c|c|c|c|}
\hline \multirow[t]{2}{*}{ Strain } & \multicolumn{2}{|c|}{$25^{\circ} \mathrm{C}-16^{\circ} \mathrm{C}$} & \multicolumn{2}{|c|}{ Heterozygote-Homozygote } \\
\hline & df & t & $\underline{\mathrm{df}}$ & 1 \\
\hline $12 \mathrm{MN}$ & 18 & $1.96 \mathrm{~ns}$ & 18 & $10.02^{* * *}$ \\
\hline $14 \mathrm{MN}$ & 18 & $8.32 * * *$ & 18 & $1.45 \mathrm{~ns}$ \\
\hline $15 \mathrm{MN}$ & 18 & $10.44 * * *$ & 18 & $6.35 * * *$ \\
\hline $16 \mathrm{MN}$ & 18 & $4.25 * * *$ & 18 & $7.29 * * *$ \\
\hline $\mathrm{Hn} 3 \mathrm{MN}$ & 18 & $5.55^{* * *}$ & 18 & $25.28 * * *$ \\
\hline dhe $3 \mathrm{MN}$ & 17 & $0.19 \mathrm{~ns}$ & 16 & $6.06 * * *$ \\
\hline hw2MN & 18 & $-4.27^{* * *}$ & 18 & $25.98 * * *$ \\
\hline
\end{tabular}

$\mathbb{d i}=$ Degrees of freedom

$\mathrm{t}=$ Values of $\mathrm{t}$ test

$* * *=\mathrm{p}<0.01$

$\mathrm{MN}$ induced mutants

tal strains for sf heteroalleles was observed while the MDT of the heterozygotes is similar to that of the mutant homozygotes.

\section{Biochemical Analyses}

The chromatographic pattern of the induced $\mathrm{mu}$ tants compared to their standards is shown in Table 5 bw $2 \mathrm{MN}$ and dke $3 \mathrm{MN}$ have a very different patterns with respect to their standards. sf $2 \mathrm{MN}$ and $\$ 6 \mathrm{MN}$ have the same pattern and sf4MN and sf5 MN have a very similar one but different from the other two. Hn $3 \mathrm{MN}$ has a decreased level of drosopterins in respect to the standard with lack of aurodrosopterin, isoxanthopterin and pterin. The quantitative estimation of the red pigment (absorbance at $480 \mathrm{~nm}$ ) and its relative percentage with respect to the wild strain Or-R, and respect to these values for the same standard mutants can be observed in Table 6. bw $2 \mathrm{MN}$ had a level of pteridines higher
Table 5. Semiquantitative estimation of pteridines in cye colour induced mutants of Drosophila melanogaster

\begin{tabular}{lcccccccc}
\hline Strain & NDP & ADP & DP & SP & ADHP & IXP & PTE & BP \\
\hline Or-R & + & + & + & + & + & + & + & + \\
bw" & 0 & 0 & 0 & 0 & 0 & 0 & 0 & 0 \\
bw2MN & -1 & -1 & -2 & + & -1 & + & 0 & +3 \\
dkest & -1 & + & -1 & + & + & + & -1 & + \\
dke3MN & + & -1 & -1 & -1 & + & -1 & -1 & +1 \\
Hn's & -1 & -2 & -1 & +2 & +1 & -1 & -1 & + \\
Hn'3MN & -2 & 0 & -2 & +1 & +1 & 0 & 0 & +1 \\
sf' & + & + & -1 & -1 & + & + & + & + \\
sf2MN & + & + & + & -1 & + & -1 & + & +1 \\
s64MN & -1 & -1 & -1 & -1 & + & -2 & + & +1 \\
st5MN & -1 & -1 & -1 & -1 & + & -1 & -1 & +1 \\
sf6MN & + & + & + & -1 & + & -1 & + & +1 \\
\hline
\end{tabular}

+ : amount approximately equal to Or-R

$+1,+2$. +3 : increasing amounts

$-1,-2$ : decreasing amounts

0 : not detected

$\mathrm{MN}$ : induced mutants

st: standard mutants

NDP, neodrosopterin; ADP, aurodrosopterin; DP, drosopterin: SP. sepiapterin: ADHP, acetyldihidrohomopterin: IXP, isoxanthopterin; PTE pterin: BP, biopterin

Table 6. Quantitative estimation of the red pigment of eyc colour and quantitative estimation of isoxanthopterin in male bodies in different standard and induced mutants of Drosopila melanogaster

\begin{tabular}{|c|c|c|c|c|}
\hline \multirow[t]{3}{*}{ Strain } & \multicolumn{2}{|c|}{ Red pigment } & \multicolumn{2}{|c|}{ Isoxanthopterin } \\
\hline & Absorbance & $\begin{array}{l}\% \text { with } \\
\text { respect }\end{array}$ & Fluorescence & $\begin{array}{l}\% \text { with } \\
\text { respect }\end{array}$ \\
\hline & at $480 \mathrm{~nm}$ & to $\mathrm{Or}-\mathrm{R}$ & & to $\mathrm{OR}-\mathrm{R}$ \\
\hline$b w^{4}$ & $0.010 \pm 0.001$ & $1.44 \pm 0.13$ & $0.9 \pm 0.2$ & $1.62 \pm 0.06$ \\
\hline $\mathrm{bw}_{2} \mathrm{MN}$ & $0.101 \pm 0.004$ & $22.52 \pm 0.42$ & $49.6 \div \hat{x} .1$ & $91.85 \pm 0.27$ \\
\hline $\mathrm{dke}^{\mathrm{st}}$ & $0.481 \pm 0.006$ & $72.92 \pm 0.91$ & $38.0 \pm 0.3$ & $68.37 \pm 0.96$ \\
\hline $\mathrm{dke} 3 \mathrm{MN}$ & $0.230 \pm 0.008$ & $51.22 \pm 0.63$ & $40.7 \pm 0.2$ & $76.32 \pm 0.73$ \\
\hline $\mathrm{Hn}^{\mathrm{rt}}$ & $0.177 \pm 0.003$ & $26.82 \pm 0.41$ & $27.9 \pm 0.4$ & $50.12 \pm 0.76$ \\
\hline $\mathrm{Hn} 3 \mathrm{MN}$ & $0.103 \pm 0.004$ & $22.94 \pm 0.15$ & $1.0 \pm 0.3$ & $1.09 \pm 0.04$ \\
\hline $\mathrm{sf}^{\mathrm{si}}$ & $0.280 \pm 0.007$ & $42.48 \pm 1.03$ & $34.5 \pm 0.5$ & $62.04 \pm 0.87$ \\
\hline sf $2 \mathrm{MN}$ & $0.238+0.005$ & $53.01 \pm 0.74$ & $45.2 \pm 0.4$ & $86.10 \pm 0.78$ \\
\hline $\mathrm{sf} 4 \mathrm{MN}$ & $0.183 \pm 0.005$ & $40.76 \pm 0.98$ & $24.0 \pm 0.2$ & $45.71 \pm 0.81$ \\
\hline sf $5 \mathrm{MN}$ & $0.182 \pm 0.007$ & $40.53 \pm 0.84$ & $35.7 \pm 0.7$ & $67.94 \pm 0.91$ \\
\hline sf6MN & $0.242 \pm 0.007$ & $53.90 \pm 0.72$ & $31.9 \pm 0.2$ & $60.90 \pm 0.82$ \\
\hline
\end{tabular}

st: standard mutants

MN: induced mutants

than the standard, aproximately $20 \%$ of the Or-R level. dke3MN and $\mathrm{Hn}^{\mathrm{r}} 3 \mathrm{MN}$ had a reduced level of pteridines in respect to the standard mutants and in sf there are two alleles (sf2MN and sf6MN) with a level of pteridines similar to the standard and 
the other two (sf4MN and sf5MN) a decreased level. The quantitative estimation of the isoxanthopterin in the induced mutants and in the standard ones is shown in Table 6 as well. The most striking is the practically lack of isoxanthopterin in $\mathrm{Hn}^{\prime} 3 \mathrm{MN}$ mutant strain compared with the standard. Opposite to this, we found the existence of isoxanthopterin in the induced bw mutant compared with the lack in the bw standard.

\section{Discussion}

Assuming that our interest was centralized in specific gene loci located in 2 nd and 3th chromosomes the specific locus test in which puntual mutations are detected through the visible phenotype, was used. Other authors with the same purpose used also this method $(5-7,12-15)$. The mutation frequencies found in this work were similar or slightly lower than that obtained by other authors for other loci $\left(5.1 \times 10^{-3}\right.$ for $\mathrm{dp},(5) ; 1.09 \times 10^{3}$ for $\mathrm{cn},(6) ; 3.7 \times 10^{4}$ for $\mathrm{Pu}$, (12). The frequencies of isolation of the mutants were also lower than those ottained by the previously mentioned authors. Most of the obtained mutants could not be isolated simply because they died before having progeny, maybe due to sterility phenomena because of the high mutagen dosis. Likewise, Paton and Sullivan (6) found that 5 of the $27 \mathrm{mu}$ tants isolated were viable as homozygotes, the remaining 22 were lethal when homozygous and were assumed to be associated with induced lethals on the second chromosome. The higher isolation frequency was obtained for sf gene. Aparisi \& Nájera (16) also found a great number of sf mutants from natural populations in respect to the frequency of the other 4 genes studied.

The high mosaicism found in this work confirms that mosaicism is a characteristic of mutagenesis with alquilant compounds. Frequencies of mosaicism for bw, dke and $\mathrm{Hn}^{\mathrm{T}}$ genes (between 30 and $42 \%$ ) are similar to those obtained for other eye colour loci as w (17), en (6) or lower than for other loci as dp (77\%) (5) since mosaicism seems more evident when mutations are induced with EMS in loci which involve a high number of cells from the ancestor to constitute the phenotypically studied organ. So, this is the most logical reason for the higher frequency found in dp locus.

EMS acted primarily on postmeiotic sperm cells, the premeiotic and meiotic cells remaining relatively inmune to its effects. In this work it was also shown that EMS had virtually no effect on premeiotic cells since only the $14.81 \%$ of the mutants appeared be- tween the 5 th-7th day after exposition and no mutant was obtained after the 7 th day.

Complementation was not found among the mutants from the same locus. This suggests the existence of only one complementation class for each one of the five studied mutations. Likewise, Paton \& Sullivan (6) do not found complementation in the production of eye colour among the cn examined mutants. Oppossite. Mackay et al. (13) found that some Pu mutants were capable to complement almost completely for viability and for defects in the eye pigments appearing some heteroallelic combinations almost wild type. Also Reynolds \& O’Donnell (7) found that some heteroallelic genotypes which allowed a low viability complemented almost completely for eye colour while others gave almost normal viabilities but low capacity to synthetize eye pigment.

The viability of the marker strain al b cn $\mathrm{sp}$ is very low and increases considerably when the EMSinduced mutation is introduced in the strain. This could be due to the removal of consanguinity in the strain when crosses were carried out in the mutant isolation scheme. In this work, the viabilities of the induced mutants at $25^{\circ} \mathrm{C}$ were higher than at $16^{\circ} \mathrm{C}$ but the phenotype was not changed. Heterozygotes showed higher viabilities than induced mutants. Other works about eye colour viabilities comparing homo- and heterozygotes in different situations (18), also showed this higher viability for heterozygous genotypes. In relation to the MDT a high variability among the alleles of the same locus and a trend to increase the MDT for the induced mutants can be observed.

The biochemical aproximation to the chromatographic pattern and to the quantitative estimation of the red pigment of the induced mutants confirms the variability found in other works (16). An interesting mutant from the biochemical point of view is bw $2 \mathrm{MN}$ with a chromatographic pattern similar to the bwV32g allele (19) but it is not lethal as in homozygosis. Its percentage of drosopterins is about $20 \%$ of the wild Or-R level while the other natural mutant and the standard have a level of about 1.2$1.4 \%$. dke $3 \mathrm{MN}$ mutant has a significatively reduced amount of red pigment with respect to the standard mutant with decreased levels of sepiapterin and isoxanthopterin compared with the mutants from the natural populations measured by Aparisi \& Nájera (16). Given that the EMS action over the genome occurs randomly, the frequency of mutation will be given so much for the size of the gene as for its importance for the viability of individual since the frequency of lethality or sterility will be higher if a mutation occurs in an important gene. 
Results obtained in this work such as the low mutagenesis frequencies for $\mathrm{Hn}^{\mathrm{r}}$ and se mutants and the fact that some metabolites are found totally absent (which seems indicative of the absolute blockade of a metabolic step) agree with the hypothesis of Bel and Ferré (1) according to which $\mathbf{H n}^{r}$ and se can be structural mutants of the pteridine pathway. This pathway in Drosophila is very complex. with steps which are not really relevant (bw, dke and sf show a great variability that do not affect life parameters) and other steps that could be thought to be structural in the pathway (se and $\mathrm{Hn}^{\prime}$ ). Nevertheless, the performance of methods to measure the enzymatic activity will be definitive to help us to discern the existence of an estructural role of both genes.

In conclusio:. : ..... out mutagenesis experiments can be it sir un wiul way to produce variability in loci we are interested and therefore a very important methodology in genetic and biochemical studies.

\section{References}

1. Bel Y. \& Ferré J. Regulation of pteridine biosynthesis and aromatic amino acid hydroxylation in Drosophila melanogaster. Biochem. Genet. 1989: 27(1/2): 59-76.

2. Ferré J, Silva FJ, Real MD. \& Ménsua JL. Pigment patterns in mutants aflecting the biosynthesis of pteridines and xanthommatin in Drosophila melanogaster. Biochem. Genet., 1986: 24(7/8): 545-569.

3. Epler JL. Ethyl methancsulfonate-induced lethals in Drosophila. Frequency-dose relations and multiple mosaicism. Genetics 1966; 54: 31-36

4. Jenkins JB. Mutagenesis at a complex locus in Drosophila with the monofunctional alkylating agent. ethyl methanesulfonate. Genetics 1967; 57: 783-793.

5. Paton DR. \& Sullivan DT. Mutagenesis at the cinnabar locus in Drosophila melanogaster. Biochem. Genet., 1978: 16(9/10): 855-865.

6. Reynolds ER. \& ODonnell JM. Characterization of new
Punch mutations: Identification of two additional mutant classes. Genetics 1988: 119: 609-617.

7. Nájera C. \& Ménsua JL. Variability of eye colour mutations in natural populations of Drosophila melanogaster. Genet. Sel. Evol. 1988; 20(1): 25-36.

8. Lewis EB. \& Bacher F. Method of feeding ethil methanesulphonate (EMS) to Drosophila males. Dros. Inf. Serv. 1968: 43: 193.

9. Robertson FW. The ecological genetics of growth in Drosophila. Growth and competitive ability of strains selected on different diets. Genet. Res. 1960; 1: 333-350.

10. Real MD, Ferré J. \& Ménsua JL. Methods for the quantitative estimation of the red and brown pigment of Drosophila melanogaster. Dros. Inf. Serv. 1985: 61: 198-199.

11. Silva FJ. \& Ménsua JL. A rapid method for quantifying isoxanthopterin in Drosophila melanogaster. Dros. Inf. Serv. 1987: 66: 166 .

12. Mackay WJ. \& O'Donnell JM. A genetic analysis of the pteridine biosynthetic enzyme. guanosine triphosphate cyclohydrolase, in Drosophila melanogaster. Genetics 1983; 105: 35-53.

13. Mackay WJ. Reynolds ER. \& O'Donnell JM. Tissue-specific and complex complementation patterns in the Punch locus of Drosophila melanogaster. Genetics 1985; 111: 885904.

14. Reynolds ER. \& O'Donnell JM. An analysis of the embryonic defects in Punch mutants of Drosophila melanogaster. Develop. Biol. 1987: 123: 430-441.

15. ODonnell JM, Boswell R, Rcynolds T. \& Mackay WJ. A cytogenetic analysis of the Punch-tudor region of chromosome 2R in Drosophila melanogaster. Genetics 1989; 121: 273-280.

16. Aparisi ML. \& Nájera C. Genctic location and biochemical characterization of eye colour mutants from natural populations of Drosophila melanogaster. Genome 1990: 33: 203-208.

17. Lee WR. Sega GA. \& Bishop JB. Chemically induced mutations observed as mosaics in Drosophila melanogaster. Mutation Res. 1970; 9: 323-336.

18. Nájera C. \& Ménsua JL. Fffect of alcohol and competition levels on viability of eye colour mutants of Drosophila melanogaster. Génct. Sél. Evol. 1985; 17(3): 331-340.

19. Lindsley DL. \& Grell EH. Genetic variation of Drosophila melanogaster. pp 42. Carnegie Inst. Washington Publ. 1968: 627. 